\title{
Three-dimensional Architecture of the Microsporidian Spore and Rapid Firing Kinetics of the Harpoon-like Invasion Machinery
}

Pattana Jaroenlak ${ }^{1}$, Michael Cammer ${ }^{1}$, Alina Davydov ${ }^{1}$, Joseph Sall ${ }^{2}$, Mahrukh Usmani ${ }^{1}$, James J. Becnel $^{3}$, Fengxia (Alice) Liang ${ }^{2}$, Damian Ekiert ${ }^{1}$ and Gira Bhabha ${ }^{1}$

${ }^{1}$ New York University School of Medicine, New York, New York, United States, ${ }^{2}$ New York University Langone Health, New York, New York, United States, ${ }^{3}$ US Department of Agriculture, Gainesville, Florida, United States

Intracellular pathogens utilize various mechanisms in order to gain entry into host cells. Microsporidia are obligate intracellular, spore-forming parasites that harness a unique and specialized harpoon-like invasion organelle called the polar tube to initiate infection. In a dormant spore, the polar tube is packaged within the spore. Under suitable environments such as high $\mathrm{pH}$ and high ionic concentrations, the polar tube shoots out of the spore as a linear tube, which the length of the tube can be approximately 30 times of its spore size. The polar tube is thought to penetrate the host cell membrane and serve as a conduit to transfer infectious cargo into the host. Microsporidian polar tube firing is one of the fastest known biological processes, and has not been well characterized. In this study, we address how the polar tube is packaged within an intact spore, the kinetics of polar tube firing in different species, and how the infectious cargo is translocated. We used serial block-face scanning electron microscopy to reveal the three-dimensional architecture of the polar tube and its relative spatial organization to other organelles. The polar tube is a right-handed coil, and is tilted relative to the anterior-posterior axis of spores. We optimized a high-speed, wide-field optical microscopy to capture and quantify the entire polar tube firing process in vitro. Our results indicate that polar tube firing kinetics differ for different species. Maximum velocity and acceleration reach approximately $300 \mu \mathrm{m} / \mathrm{s}$ and $6,600 \mu \mathrm{m} / \mathrm{s}^{2}$, respectively. As polar tube firing happens, cargo begins to travel through the polar tube. We used nucleus as a marker and observed that the nucleus, which is many times the size of the polar tube diameter, undergoes vast deformation to fit into the narrow polar tube, and moves at speeds faster than polar tube firing. Our study sheds new lights on the structure, dynamics of the polar tube firing, and unravels how cargo moves through the narrow tube. 

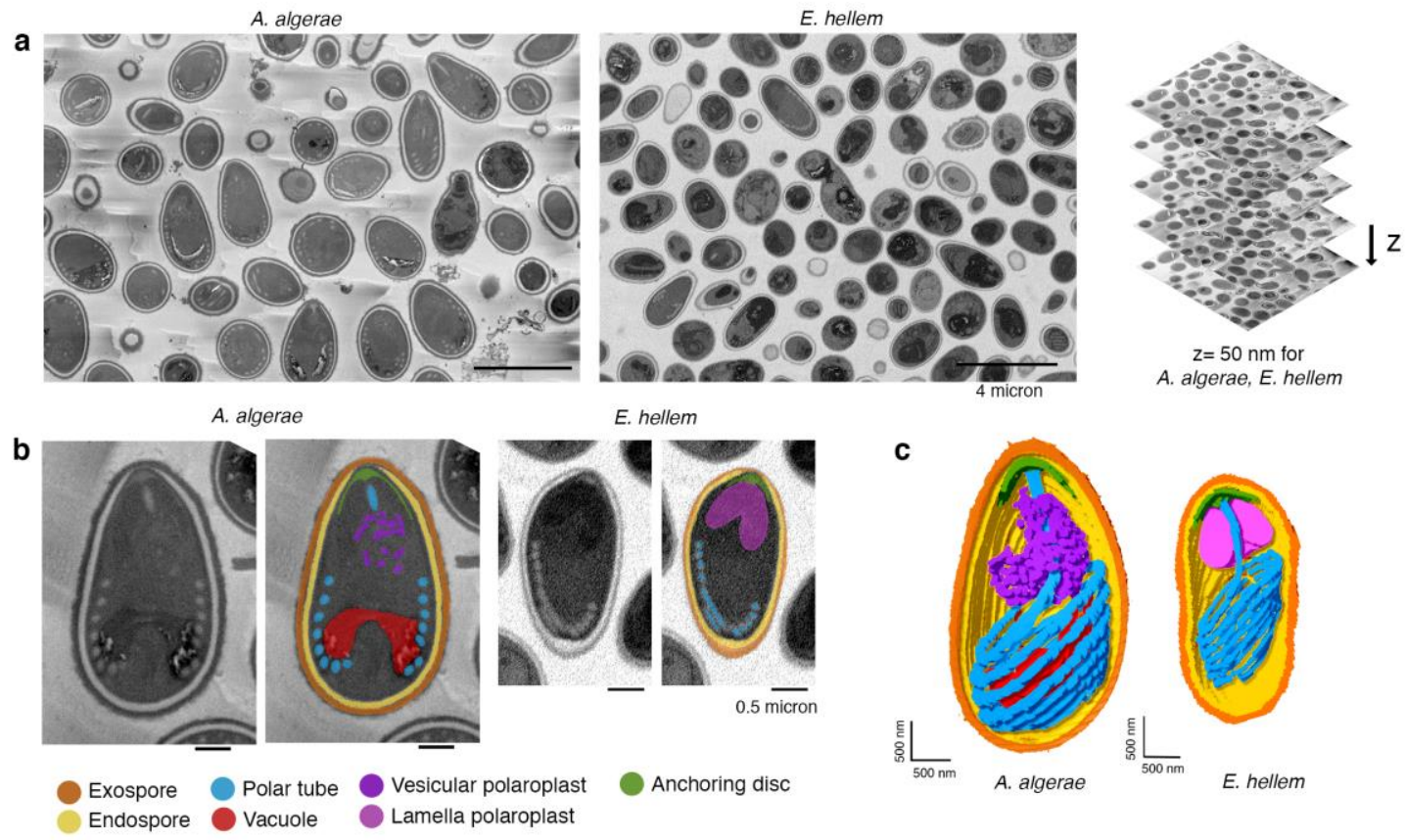

Figure 1. Spatial organization of the polar tube and other organelles in an intact spore. (a) Serial-block face SEM sections of Anncaliia algerae and Encephalitozoon hellem intact spores. The thickness of the sections is $50 \mathrm{~nm}$. The dataset was collected to cover the whole spores. (b) Segmentation of organelles in the spore. Each color represents different organelles. Color keys are showed in the figure. (c) 3D reconstruction of $A$. algerae and $E$. hellem spores reveal overall architecture of the polar tube.

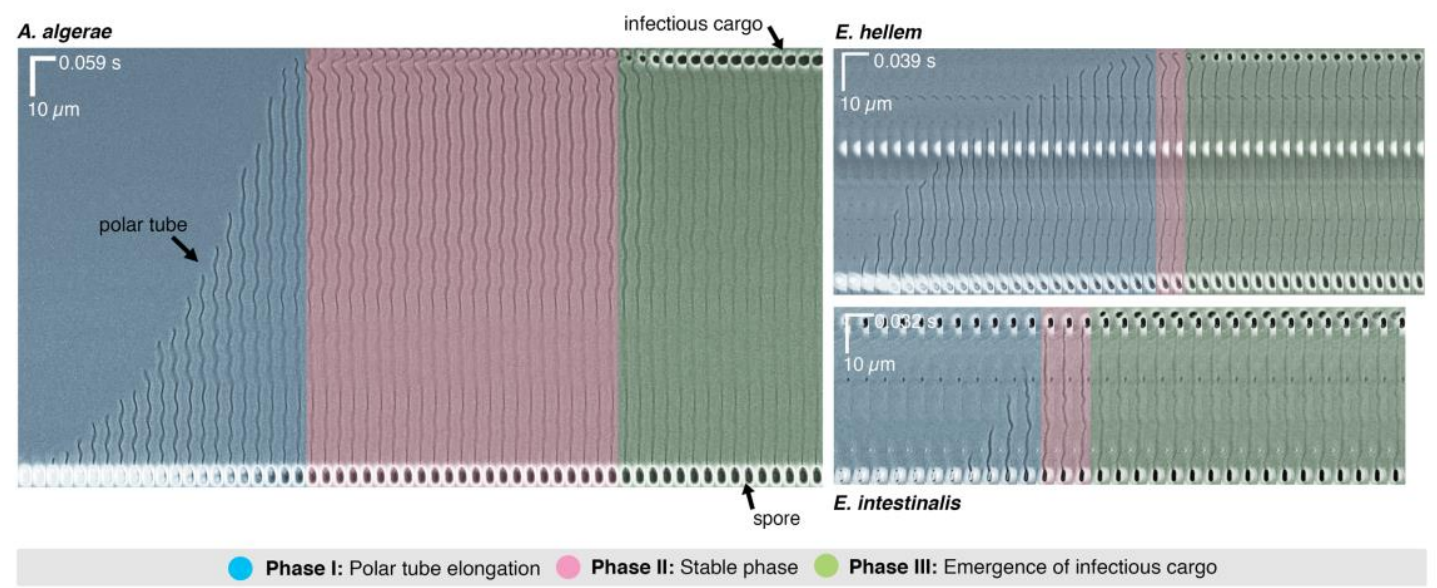

Figure 2. Figure 2: Polar tube firing kinetics differ across microsporidia genera. Kymographs of the polar tube firing from A. algerae (Left), E. hellem (Top-right), and E. intestinalis (Bottom-right). Time interval and polar tube length are showed in the $\mathrm{X}$-axis and $\mathrm{Y}$-axis of the scale bar, respectively. The polar tube firing process can be divided into 3 phases, namely elongation phase, stable phase, and emergence of infectious cargo phase. References

Frixione, E. et al. Dynamics of polar filament discharge and sporoplasm expulsion by microsporidian spores. Cell Motility and the Cytoskeleton vol. 22 38-50 (1992). 
Weiss, L. M. \& Becnel, J. J. Microsporidia: Pathogens of Opportunity. (John Wiley \& Sons, 2014). 